\title{
Cytophilic antibodies to Plasmodium falciparum Glutamate Rich Protein are associated with malaria protection in an area of holoendemic transmission
}

\author{
John PA Lusingu*1,2, Lasse S Vestergaard ${ }^{2,3}$, Michael Alifrangis², \\ Bruno P Mmbando ${ }^{1}$, Michael Theisen ${ }^{3}$, Andrew Y Kitua ${ }^{1}$, Martha M Lemnge ${ }^{1}$ \\ and Thor G Theander ${ }^{2}$
}

\begin{abstract}
Address: ${ }^{1}$ National Institute for Medical Research, Amani Medical Research Centre, Tanga \& Headquarters, Dar es Salaam, Tanzania, ${ }^{2}$ Centre for Medical Parasitology, Institute of Medical Microbiology and Immunology, University of Copenhagen and Department of Infectious Diseases, Copenhagen University Hospital (Rigshospitalet), Denmark and ${ }^{3}$ Statens Serum Institut, Copenhagen, Denmark

Email: John PA Lusingu* - jpalusingu@yahoo.co.uk; Lasse S Vestergaard - lsv@dadlnet.dk; Michael Alifrangis - alifrangis@cmp.dk; Bruno P Mmbando - pbruno@amani.mimcom.net; Michael Theisen - MTH@ssi.dk; Andrew Y Kitua - akitua@nimr.or.tz; Martha M Lemnge-mlemnge@amani.mimcom.net; Thor G Theander - theander@cmp.dk

* Corresponding author
\end{abstract}

Published: 29 September 2005

Malaria Journal 2005, 4:48 doi:10.1 186/1475-2875-4-48

This article is available from: http://www.malariajournal.com/content/4/I/48

(C) 2005 Lusingu et al; licensee BioMed Central Ltd.

This is an Open Access article distributed under the terms of the Creative Commons Attribution License (http://creativecommons.org/licenses/by/2.0), which permits unrestricted use, distribution, and reproduction in any medium, provided the original work is properly cited.
Received: 08 June 2005

Accepted: 29 September 2005

\begin{abstract}
Background: Several studies conducted in areas of medium or low malaria transmission intensity have found associations between malaria immunity and plasma antibody levels to glutamate rich protein (GLURP). This study was conducted to analyse if a similar relationship could be documented in an area of intense malaria transmission.
\end{abstract}

Methods: A six month longitudinal study was conducted in an area of holoendemic malaria transmission in north-eastern Tanzania, where the incidence of febrile malaria decreased sharply by the age of three years, and anaemia constituted a significant part of the malaria disease burden. Plasma antibodies to glutamate rich protein (GLURP) were analysed and related with protection against malaria morbidity in models correcting for the effect of age.

Results: The risk of febrile malaria episodes was reduced significantly in children with measurable anti-GLURP IgGI antibodies at enrolment [adjusted odds ratio: $0.39(95 \% \mathrm{Cl}: 0.15,0.99) ; P=$ 0.047]. Interestingly, there was an inverse relationship between the plasma anti-GLURP IgGI and IgG3 levels and the levels of parasitaemia at enrolment. However, anti-GLURP IgG2 and IgG4 levels were not associated with reduction in parasite density. Similarly, antibody levels were not associated with haemoglobin levels or anaemia risk.

Conclusion: Cytophilic IgGI and IgG3 antibodies against R0-GLURP may contribute to the control of parasite multiplication and reduction in febrile malaria incidence in children living in an area of intense malaria transmission. 


\section{Background}

In areas of stable malaria transmission, immunity is acquired during childhood $[1,2]$, and the protection is mainly mediated by antibodies directed against the blood stages of the parasite [3]. The relationship between malaria morbidity and antibody levels to malaria antigens has been analysed in several prospective longitudinal studies performed in different parts of Africa and Asia [49]. The Glutamate Rich Protein (GLURP) is a Plasmodium falciparum antigen, which has been studied extensively. It is a $220 \mathrm{kD}$ protein expressed in the hepatic, asexual and sexual stages of the parasite life cycle [10]. The protein can be divided into an N-terminal non-repeat region $\left(\mathrm{R}_{25-500}\right.$ or R0), a central repeat region (R1) and a C-terminal repeat region (R2) [11]. GLURP is a malaria vaccine candidate, which has undergone phase 1 trials in Europe and trials are planned to take place in Africa in the near future.

Several immuno-epidemiological studies using sera and clinical data from various sites have consistently identified high anti-R0-GLURP immunoglobulin G (IgG) levels as significant predictors of protection against high levels of parasitaemia, and febrile malaria episodes [6,12-16]. The protective antibodies are thought to elicit antibody dependent cytotoxic inhibition (ADCI) [17] through binding to the surfaces of merozoites [18]. Most of these studies have been performed in areas of moderate malaria transmission where protection against malaria fevers is achieved in those aged 5-15 years. In this report, plasma antibody levels to R0-GLURP was measured and related to malaria morbidity in a village subjected to holoendemic transmission and entomological inoculation rates exceeding one infectious bite per night [2]. In this community the incidence of febrile malaria decreases sharply by the age of three years and anaemia constitutes a significant part of the malaria disease burden [19]. Antibody levels to R0-GLURP in two other villages located in areas of moderate and low transmission were measured to compare the age related acquisition of antibodies in individuals living under different malaria transmission intensity.

\section{Materials and methods Study sites and population}

A longitudinal malariometric study was carried out in three villages with different malaria transmission intensity in the Tanga region, Tanzania, as described in detail elsewhere [19]. The villages are situated at varying altitudes, which in north-eastern Tanzania is a proxy for malaria transmission intensity [20]. Malariometric surveys were conducted and blood samples were collected in April, July and September. Haemoglobin levels were measured using a HemoCue ${ }^{\circledast}$ photometer (Ångelholm, Sweden) and thick and thin blood smears for malarial microscopy were prepared. Thereafter, blood was centrifuged to obtain plasma, which was frozen at $-20^{\circ} \mathrm{C}$. Local village helpers and health workers at nearby health facilities performed passive case detection during the six month study period. The village helpers were provided with first-line antimalarial drug (sulphadoxine-pyrimethamine), paracetamol, microscope slides, blood lancets, treatment charts, febrile case detection forms and storage boxes. Villagers could seek treatment at any time from these helpers. Patients with symptoms of malaria were treated with the first-line antimalarial drug. If they had severe symptoms or did not respond adequately to the first-line treatment, they were referred to a health facility. Prior to treatment, the village helpers collected clinical information and a malaria blood smear. At each nearby health facility, two permanent staff members monitored study participants seeking medical treatment at the facility. If study participants presented at the facility with a history of fever, a form was completed and a blood smear collected. Active febrile case detection was undertaken once per month by the research team. During active case detection, study participants were seen by a trained physician and a blood smear was taken from all study participants who had reported a history of fever within two days and/or had axillary temperature $\geq$ $37.5^{\circ} \mathrm{C}$.

\section{Case definitions and selection of plasma samples for antibody assays}

Febrile malaria episodes were defined as an axillary temperature $\geq 37.5^{\circ} \mathrm{C}$ and/or a history of fever within the previous 48 hours in the presence of asexual $P$. falciparum parasites $\geq 5000$ parasites/ $\mu \mathrm{l}$ [19]. Anaemia was defined as haemoglobin $<11.0 \mathrm{~g} / \mathrm{dl}[21]$.

The incidence of febrile malaria episodes was low in Magamba and Ubiri [19]. In Mgome, 219 of the 254 individuals completed the longitudinal follow-up and from 171 of these individuals, sufficient plasma was available to measure R0-GLURP IgG class and subclass levels. Thus, antibody levels were measured in 9, 24, 33, 52, 35, and 18 individuals belonging to the 0-11 months, $1-2$ years, 34 years, 5-9 years, 10-14 years, and 15-19 years age groups, respectively. Of the 171 individuals, 54 had febrile malaria episodes and 44 developed anaemia during the follow-up period.

To compare age-specific acquisition of R0-GLURP IgG class antibodies in areas of different endemicity, plasma samples of 40 individuals from Ubiri village (moderate transmission) and Magamba village (low transmission) were also tested. The samples were selected randomly from asymptomatic individuals to represent four age groups (0-4, 5-9, 10-14 and 15-19 years, $\mathrm{N}=10$ in each group). Since malaria morbidity was low in Ubiri and Magamba, no attempt was made to relate morbidity and anti-R0-GLURP IgG levels in these villages. 


\section{Antibody assays}

Antibodies to R0-GLURP were measured by enzymelinked immunosorbent assay (ELISA) based on a protocol developed by Afro Immunoassay. Briefly, microtitre plates (Maxisorp Nunc, Roskilde, Denmark) were coated overnight at $4{ }^{\circ} \mathrm{C}$ with purified his-tag produced recombinant R0-GLURP $(0.5 \mu \mathrm{g} / \mathrm{ml})$ diluted in phosphate buffered saline (PBS). The plates were blocked with $3 \%$ powderedmilk-containing-phosphate buffer for one hour. Plasma from samples diluted 1:200 in dilution buffer (PBS with $1 \%$ powdered-milk and $0.1 \%$ Tween-20) was added in duplicate. The plates were then incubated at room temperature for one hour, where after peroxidase-conjugated rabbit anti-human IgG or IgM (Dako, Glostrup, Denmark) was added. Plates were washed four times with washing buffer (PBS with 0.1\% Tween-20 and $0.5 \mathrm{M}$ $\mathrm{NaCl}$ ) between steps. Colour was developed using hydrogen peroxide with $\mathrm{O}$-phenylenediamine (Dako, Glostrup, Denmark), and reading of antibody absorbance was done at $492 \mathrm{~nm}$. Samples were retested if the measured differences in absorbance values between duplicate samples were higher than $15 \%$. For determination of IgG subclasses (IgG1, IgG2, IgG3 and IgG4), plasma samples diluted 1:50 were added in duplicate and incubated for one hour at room temperature. The following monoclonal mouse anti-human subclasses were used: clone NL16 for IgG1 (Sky lab), clone NP6002 for IgG2 (Sigma), clone ZG4 for IgG3 (Sky lab) and clone RJ4 for IgG4 (Sky lab). The monoclonal IgG subclasses were diluted 1:2000 for IgG1, 1:3000 for IgG2, IgG3 and IgG4 in dilution buffer and incubated for one hour at room temperature. Goat anti-mouse IgG conjugated to peroxidase (Caltag) diluted 1:3000 in dilution buffer was then added and plates incubated for one hour. Colour development and reading of antibody absorbance was done as described above. Antibody levels were measured relative to the titration of IgG and IgG1-4 standard solutions. Plasma samples from 31 adult healthy Danes without any previous exposure to malaria were used as negative controls to generate cut-off values, which was defined as mean plus two standard deviations for the respective IgG class or subclasses. A positive control containing a pool of plasma from adult Liberian individuals (kindly provided by Dr. S. Jepsen, Statens Serum Institut, Copenhagen, Denmark through Afro Immuno Assay) were tested in parallel to the study samples. The positive plasma pool contained 27.7, $48.7,35.7,18.5$, and 46.6 arbitrary units (AU)/ml antiR0GLURP antibodies of IgG, IgG1, IgG2, IgG3, and IgG4, respectively. The cut-off values in arbitrary units were 3.5, $1.4,1.4,1.7$, and $0 \mathrm{AU} / \mathrm{ml}$ for IgG, IgG1, IgG2, IgG3, and IgG4, respectively. The highest OD values in positive samples were 2.7 for IgG and 3.8 for IgG1-4.

\section{Data analysis}

Data were analysed using Stata/SE version 8.2 (Stata Corporation, Texas, USA; http://www.stata.com). The age dependence of antibody responses was analyzed by use of Spearman's rank-order correlation and the mean matched-pairs test was used to compare R0-GLURP IgG levels between samples collected at the initiation and at the end of the study. The Mann-Whitney test was used to test R0-GLURP IgG class and subclasses levels between females and males. Univariate and multivariate models were fitted to estimate to what extent R0-GLURP IgG class and subclass levels could be attributed to protection against high parasite density, febrile malaria episodes or anaemia in individuals from the high transmission village. Differences were considered statistically significant if the $95 \%$ confidence interval was not overlapping or $P<$ 0.05 .

\section{Results \\ Plasma antibody levels to R0-GLURP in areas of different malaria endemicity}

The prevalence and mean levels of anti-R0-GLURP IgG were highest in residents living under intense and holoendemic malaria transmission in Mgome, lower in individuals living under moderate and seasonal transmission in Ubiri and lowest in residents living under low and unstable transmission in Magamba (Figure 1). The differences in IgG levels between these villages were highly significant $(P(z)<0.001$, Trend test $)$. The prevalence and levels of antibodies increased with donor age in Mgome and Ubiri villages (Figure 1), although there was a tendency of slight decrease in antibody levels after the age of 14 years in Mgome. Antibody levels did not differ significantly between males and females (Mann-Whitney test Mgome $\mathrm{P}(\mathrm{z})=0.40$; Ubiri $\mathrm{P}(\mathrm{z})=0.12$.; Magamba $\mathrm{P}(\mathrm{z})=0.94)$. In Mgome, there was no statistical difference in antibody levels between samples collected at the beginning and at the end of the study (mean difference [95\% confidence interval $(\mathrm{CI})]$ : 4.0 arbitrary units (AU) $[-15.8,23.9], \mathrm{P}(\mathrm{t})=$ 0.68 , paired T-test). The mean antibody levels were slightly higher in samples collected at the end of the study in Ubiri (mean difference [95\%CI]: 6.4 AU [-2.1, 15.0], $\mathrm{P}(\mathrm{t})=0.13$, paired T-test) and Magamba (mean difference [95\%CI]: 7.6 AU $[6.2,9.0], \mathrm{P}(\mathrm{t})<0.0001$, paired T-test).

In all three villages, R0-GLURP IgM was detected at very low levels with no distinct patterns observed with respect to age, sex or season (data not shown).

\section{IgG subclass responses to R0-GLURP in Mgome village}

The prevalence and mean levels of R0-GLURP IgG antibodies increased with age for all subclasses. For the cytophilic IgG1 and IgG3, there was a marked increase in three to four year old children (Figure 2). For the noncytophilic IgG2 and IgG4, there was a steady increase in 


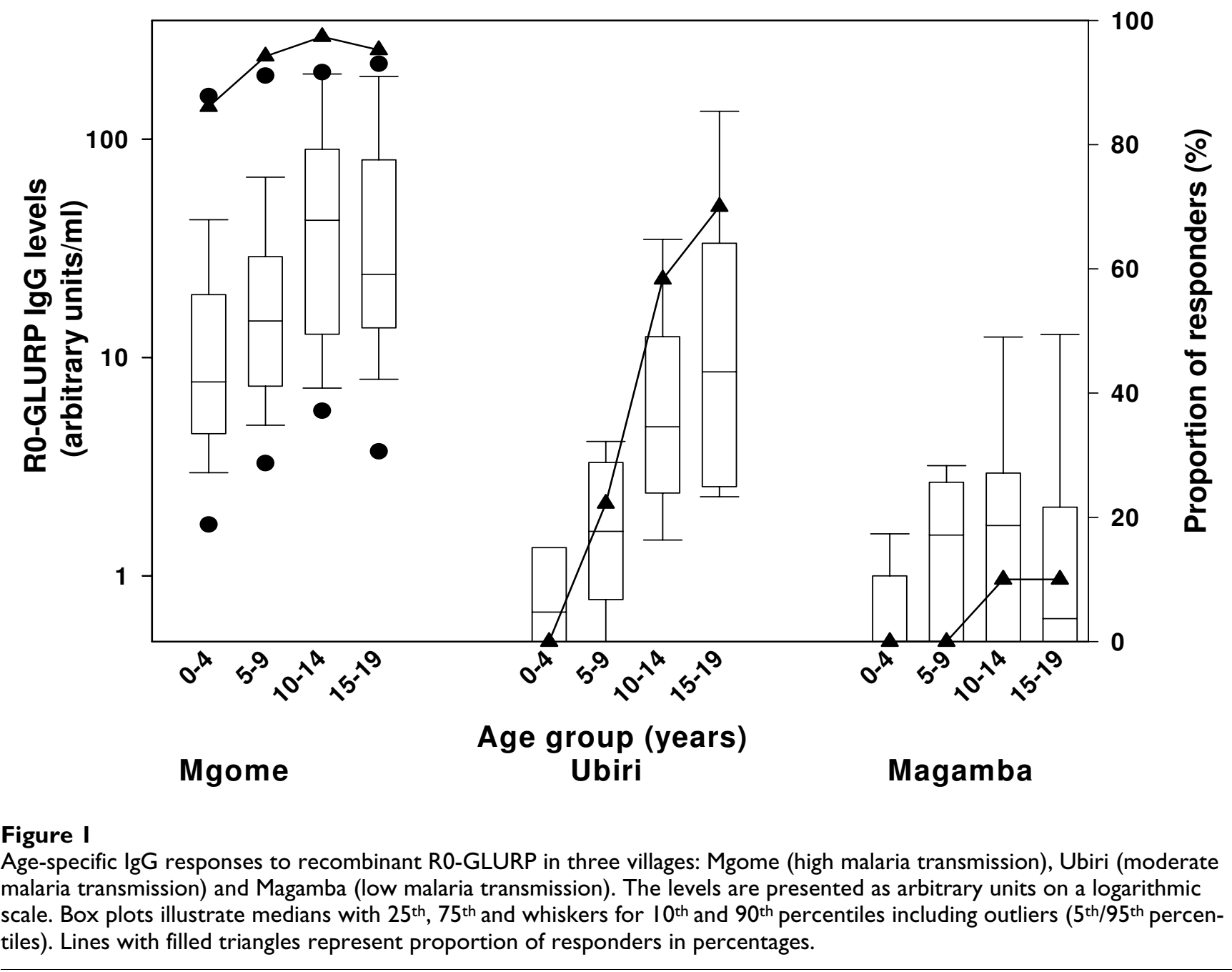

the levels and proportion of responders throughout the age groups (Figure 2). The levels of IgG4 antibodies were generally low.

\section{Relationship between R0-GLURP IgG levels and Plasmodium falciparum density in Mgome (high transmission village)}

Multiple linear regression models adjusting for age were generated to determine whether the anti-R0-GLURP IgG levels were associated with $P$. falciparum density at the initiation of the study. Interestingly, there was a significant association between cytophilic IgG subclass antibodies and reduced parasite density. For IgG1, one $\log$ unit increase in antibodies was associated with a reduction in $P$. falciparum density by 1,401 parasites/ $\mu$ l [95\% CI: 231 ,
2571; $P=0.019]$; and for IgG3 level, one log unit increase was associated with a reduction in $P$. falciparum density at enrolment by 983 parasites/ $\mu$ [ [95\% CI: 126, $1841 ; P=$ 0.025]. Although similar associations were observed in univariate analysis for non-cytophilic IgG subclasses such associations were not significant after adjusting for age (Table 1).

\section{Relationship between R0-GLURP IgG and risk of developing febrile malaria in Mgome}

The risk of developing a febrile malaria episode during the study decreased with age and was $65.2 \%(43 / 66), 11.5 \%$ $(6 / 52), 11.4 \%(4 / 37)$ and $5.6 \%(1 / 18)$ for the age groups 0-4, 5-9, 10-14 and 15-19, respectively. Logistic regression models correcting for age indicated that the presence 
A
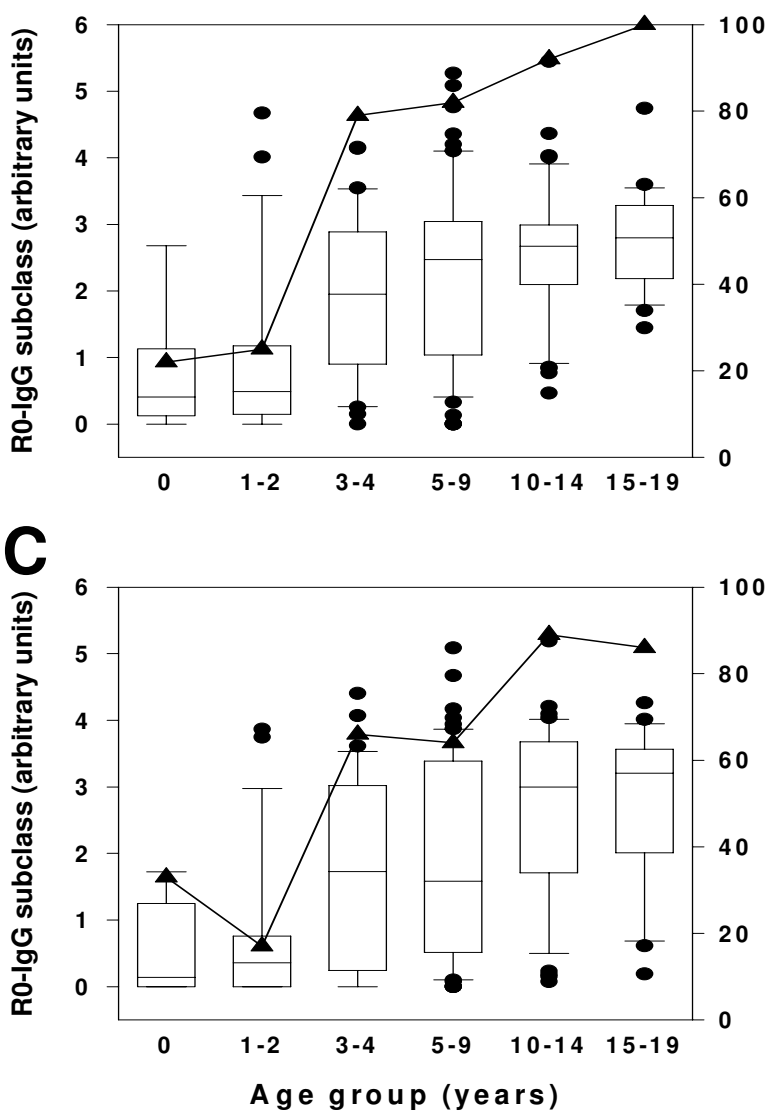

B

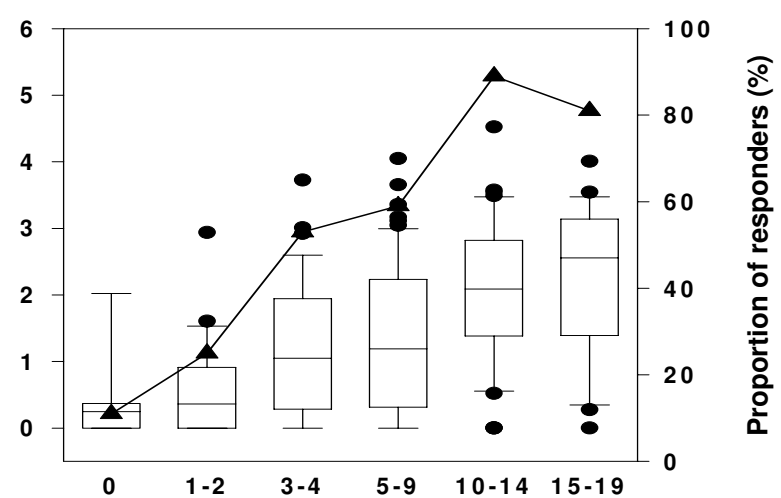

D

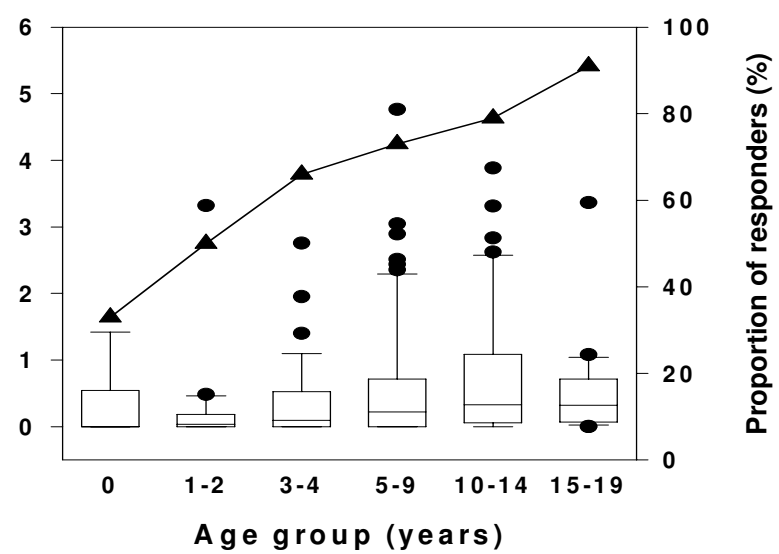

Figure 2

Age-specific lgG subclass responses to recombinant R0-GLURP in Mgome (high transmission village). Panels A, B, C and D represent $\lg \mathrm{I}, \lg 2$, IgG3, and $\lg G 4$ subclasses, respectively. The levels are presented as arbitrary units on a logarithmic scale. Box plots illustrate medians with $25^{\text {th }}, 75^{\text {th }}$ and whiskers for $10^{\text {th }}$ and $90^{\text {th }}$ percentiles including outliers for each $\lg G$ subclass. Lines with filled triangles represent prevalence of responders in percentages.

of a measurable R0-GLURP IgG1 was associated with a reduced risk of febrile malaria episodes [adjusted odds ratio (AOR) 0.39 (95\% CI: $0.15,0.99), P=0.047]$. The age adjusted odds ratio for individuals who had a measurable IgG3 response was 0.52 , but the $95 \%$ confidence interval for this estimate was wide and not significantly different from one (Table 2). The presence of antibodies to R0GLURP of the IgG2 or IgG4 subclasses was not associated with significant reduction in the risk of febrile malaria episodes.

\section{Relationship between R0-GLURP IgG and risk of anaemia in Mgome}

In logistic regression models including age and sex, there were no statistically significant associations between the risk of anaemia and having a measurable IgG [AOR: 1.03 $(0.45,2.35) ; P=0.95]$ or IgG subclass (data not shown) R0-GLURP antibody response. Similarly, in linear regression models correcting for age and sex, there were no associations between plasma R0-GLURP IgG or IgG subclass levels and the haemoglobin level at enrolment (data not shown).

\section{Discussion}

Studies in which observed clinical protection is linked to the level of malaria antibodies on an individual level have been used to identify vaccine targets $[5,6,15,22]$. In these types of studies, participants are often divided into susceptible and protected individuals and this obviously requires that a reasonable number of the participants 
Table I: Relationship between P. falciparum density (parasites/ $\mu$ I) and anti R0-GLURP IgG levels (log arbitrary unit/ml) at enrolment.

\begin{tabular}{|c|c|c|c|c|}
\hline R0-GLURP antibodies & $\begin{array}{l}\text { Unadjusted coefficients } \\
(95 \% \mathrm{Cl})^{\prime}\end{array}$ & $P$-value & $\begin{array}{l}\text { Adjusted coefficients ( } 95 \% \\
\mathrm{Cl})^{2}\end{array}$ & $P$-value \\
\hline $\lg G$ & $-773(-805,689)$ & 0.378 & $-146(-533,3457)$ & 0.15 \\
\hline $\lg G \mid$ & $-2136(-3477,-795)$ & 0.002 & $-|40|(-257|,-23|)$ & 0.019 \\
\hline $\lg G 2$ & $-1512(-2894,-128)$ & 0.032 & $-719(-2135,697)$ & 0.318 \\
\hline $\lg G 3$ & $-1482(-2542,-423)$ & 0.006 & $-983(-1841,-126)$ & 0.025 \\
\hline $\lg G 4$ & $58(-590,706)$ & 0.86 & $195(-436,826)$ & 0.542 \\
\hline
\end{tabular}

I Univariate linear regression. ${ }^{2}$ Multiple linear regressions adjusting for the effect of age including age as square root of age. Models using other forms of age correction (age, age $\times$ age, log age) gave similar results.

Table 2: Odds ratio for the risk of febrile malaria episodes

\begin{tabular}{lcccc}
\hline R0-GLURP antibodies & $\begin{array}{l}\text { Unadjusted odds ratio } \\
(95 \% \mathrm{Cl}){ }^{\prime}\end{array}$ & $\begin{array}{l}\text { P-value } \\
\text { Clj) }\end{array}$ & $\begin{array}{l}\text { Adjusted odds ratio }(95 \% \\
\text { C-value }\end{array}$ \\
\hline $\lg G$ & $0.49(0.25-0.94)$ & 0.031 & $1.13(0.50-2.53)$ & 0.77 \\
$\operatorname{lgGI}$ & $0.17(0.07-0.42)$ & $<0.001$ & $0.39(0.15-0.99)$ & 0.047 \\
$\operatorname{lgG2}$ & $0.47(0.20-1.08)$ & 0.075 & $1.42(0.58-3.46)$ & 0.445 \\
$\operatorname{lgG3}$ & $0.25(0.11-0.58)$ & 0.001 & $0.52(0.20-1.40)$ & 0.197 \\
$\operatorname{lgG} 4$ & $0.42(0.18-0.99)$ & 0.046 & $0.77(0.30-2.03)$ & 0.602 \\
\hline
\end{tabular}

I Logistic regression indicating risk of febrile malaria episode in individuals with detectable plasma levels of anti RO-GLURP antibodies (for lgG, IgG I, $\lg 2$, IgG3 and lgG4, respectively) relative to those without a measurable antibody response. ${ }^{2}$ Adjusted for the effect of age including age as square root of age.

develop clinical symptoms during follow up. To allow comparisons between immunological parameters, it is also preferable that the protected individuals are as closely age matched to the susceptible individuals as possible. Due to problems of obtaining sufficient amounts of plasma from infants and young children, most studies have been conducted in areas of moderate transmission targeting children between 5-15 years or in areas of high transmission targeting children over 5 years and adults. These studies have documented that the presence of R0GLURP IgG is associated with protection against febrile malaria attacks in areas of moderate and seasonal malaria transmission [6,13-15]. The acquisition of malaria immunity is governed by the transmission intensity [23], and in areas of holoendemic transmission children of three to four years of age have already developed considerable protection against febrile episodes $[14,15]$. In the current study, individuals with a measurable anti R0-GLURP IgG1 response had a statistically significant reduction in the risk of getting a febrile malaria attack compared with those without such antibodies. The results cannot unravel whether GLURP antibodies directly were responsible for the effect or whether they constitute a marker for other immunological activities. The protection provided by GLURP antibodies is mainly thought to be mediated through IgG1 and IgG3 antibodies which dampen the growth of blood stage parasites by antibody dependent cellular inhibition (ADCI) $[13,14,17,24]$; although levels of IgG2 GLURP antibodies have also been implicated in protection $[13,14]$. It is, therefore, of interest that a significant association between the level of anti R0-GLURP IgG1 and IgG3 antibodies and the parasite density at enrolment has been found in this study.

The acquisition of IgG against GLURP was highly dependant on malaria transmission intensity. In the high transmission village antibody levels increased markedly after the age of two years, and in the three to four years old children a very high percentage had a detectable antibodies response. In the village with moderate transmission antibody acquisition was much slower and GLURP antibody response rates over 50\% were only seen in the age groups older than 10 years.

In areas of high malaria transmission one of the major disease burdens attributable to $P$. falciparum infection is anaemia occurring in infants and young children $[2,19,21]$. In Mgome, all children under two years had haemoglobin levels under $11 \mathrm{~g} / \mathrm{dl}$. Thus, it was disappointing that the levels of anti R0-GLURP antibodies were 
not associated with haemoglobin levels in multiple linear regression models or with anaemia in multiple logistic regression models.

\section{Conclusion}

In conclusion, this study conducted in an area of intense malaria transmission detected an association between protection against febrile malaria disease and presence of anti R0-GLURP antibodies and indicated that increasing levels of antibodies of the IgG1 and the IgG3 subclasses are associated with a reduction in $P$. falciparum parasite densities.

\section{Authors' contributions}

JPAL carried out field surveys, performed ELISA, analysed data and drafted the manuscript. LSV carried out field surveys and in collaboration with JPAL, MA and MT contributed to the set up of the ELISA. BPM carried out field surveys and participated in data analysis. MT, AYK, MML and TGT conceived the design of the study. All authors amended and approved the final manuscript.

\section{Acknowledgements}

We are grateful to all study participants including their parents/guardians as well as village helpers and health management teams in Tanga region. We acknowledge with thanks Anne Corfitz, Juma Akida, Zacharia Savael, Susanne Pedersen, Jimmy Weng, Fabio-Avit Massawe, John Hiza, William Chambo, Donald Mwanjeluka and Seth Nguhu for excellent technical assistance throughout the study. We are grateful to Drs Lars Hviid, Thomas Scheike and Daniel Dodoo for several suggestions and advice during the analysis. The study was conducted under the auspices of the Joint Malaria Programme, a collaborative research initiative between the Centre for Medical Parasitology at the University of Copenhagen and Copenhagen University Hospital, the Kilimanjaro Christian Medical College, the London School of Hygiene and Tropical Medicine and the Tanzania National Institute for Medical Research. John Lusingu is supported by a PhD scholarship from the Gates Malaria Partnership. The study was also supported by the ENRECA programme of the Danish International Development Agency (DANIDA).

\section{References}

I. Marsh K, Snow RW: Host-parasite interaction and morbidity in malaria endemic areas. Philos Trans R Soc Lond B Biol Sci 1997, 352:1385-1394.

2. Ellman R, Maxwell C, Finch R, Shayo D: Malaria and anaemia at different altitudes in the Muheza district of Tanzania: childhood morbidity in relation to level of exposure to infection. Ann Trop Med Parasitol 1998, 92:741-753.

3. Sabchareon A, Burnouf T, Ouattara D, Attanath P, Bouharoun-Tayoun $\mathrm{H}$, Chantavanich P, Foucault C, Chongsuphajaisiddhi T, Druilhe P: Parasitologic and clinical human response to immunoglobulin administration in falciparum malaria. Am J Trop Med Hyg |991, 45:297-308.

4. Riley EM, Allen SJ, Wheeler JG, Blackman MJ, Bennett S, Takacs B, Schonfeld HJ, Holder AA, Greenwood BM: Naturally acquired cellular and humoral immune responses to the major merozoite surface antigen (PFMSPI) of Plasmodium falciparum are associated with reduced malaria morbidity. Parasite Immunol 1992, 14:321-337.

5. Conway DJ, Cavanagh DR, Tanabe K, Roper C, Mikes ZS, Sakihama N, Bojang KA, Oduola AM, Kremsner PG, Arnot DE, Greenwood $B M$, McBride JS: A principal target of human immunity to malaria identified by molecular population genetic and immunological analyses. Nat Med 2000, 6:689-692.

6. Soe S, Theisen M, Roussilhon C, Aye KS, Druilhe P: Association between protection against clinical malaria and antibodies to merozoite surface antigens in an area of hyperendemicity in Myanmar: complementarity between responses to merozoite surface protein 3 and the 220-kilodalton glutamaterich protein. Infect Immun 2004, 72:247-252.

7. Dodoo D, Theander TG, Kurtzhals JA, Koram K, Riley E, Akanmori $B D$, Nkrumah FK, Hviid L: Levels of antibody to conserved parts of Plasmodium falciparum merozoite surface protein I in Ghanaian children are not associated with protection from clinical malaria. Infect Immun 1999, 67:2131-2137.

8. Bull PC, Lowe BS, Kortok M, Molyneux CS, Newbold Cl, Marsh K: Parasite antigens on the infected red cell surface are targets for naturally acquired immunity to malaria. Nat Med 1998, 4:358-360.

9. Giha HA, Staalsoe T, Dodoo D, Roper C, Satti GM, Arnot DE, Hviid L, Theander TG: Antibodies to variable Plasmodium falciparuminfected erythrocyte surface antigens are associated with protection from novel malaria infections. Immunol Lett 2000 , 71:117-126.

10. Dziegiel M, Borre MB, Jepsen S, Hogh B, Petersen E, Vuust J: Recombinant Plasmodium falciparum glutamate rich protein; purification and use in enzyme-linked immunosorbent assay. Am Trop Med Hyg 1991, 44:306-313.

II. Theisen M, Vuust J, Gottschau A, Jepsen S, Hogh B: Antigenicity and immunogenicity of recombinant glutamate-rich protein of Plasmodium falciparum expressed in Escherichia coli. Clin Diagn Lab Immunol 1995, 2:30-34.

12. Hogh B, Petersen E, Dziegiel M, David K, Hanson A, Borre M, Holm $A$, Vuust J, Jepsen S: Antibodies to a recombinant glutamaterich Plasmodium falciparum protein: evidence for protection of individuals living in a holoendemic area of Liberia. Am J Trop Med Hyg 1992, 46:307-3I 3.

13. Dodoo D, Theisen M, Kurtzhals JA, Akanmori BD, Koram KA, Jepsen S, Nkrumah FK, Theander TG, Hviid L: Naturally acquired antibodies to the glutamate-rich protein are associated with protection against Plasmodium falciparum malaria. J Infect Dis 2000, I 8 I: | 202-1205.

14. Oeuvray C, Theisen M, Rogier C, Trape JF, Jepsen S, Druilhe P: Cytophilic immunoglobulin responses to Plasmodium falciparum glutamate-rich protein are correlated with protection against clinical malaria in Dielmo, Senegal. Infect Immun 2000, 68:2617-2620.

15. Meraldi V, Nebie I, Tiono AB, Diallo D, Sanogo E, Theisen M, Druilhe $P$, Corradin G, Moret R, Sirima BS: Natural antibody response to Plasmodium falciparum Exp-I, MSP-3 and GLURP long synthetic peptides and association with protection. Parasite Immunol 2004, 26:265-272.

16. Dziegiel M, Rowe P, Bennett S, Allen SJ, Olerup O, Gottschau A, Borre $M$, Riley $E M$ : Immunoglobulin $M$ and $G$ antibody responses to Plasmodium falciparum glutamate-rich protein: correlation with clinical immunity in Gambian children. Infect Immun 1993, 6 I:103-108.

17. Bouharoun-Tayoun H, Oeuvray C, Lunel F, Druilhe P: Mechanisms underlying the monocyte-mediated antibody-dependent killing of Plasmodium falciparum asexual blood stages. J Exp Med 1995, I 82:409-4I8.

18. Theisen M, Soe S, Oeuvray C, Thomas AW, Vuust J, Danielsen S, Jepsen S, Druilhe P: The glutamate-rich protein (GLURP) of Plasmodium falciparum is a target for antibody-dependent monocyte-mediated inhibition of parasite growth in vitro. Infect Immun 1998, 66: I I-17.

19. Lusingu JP, Vestergaard LS, Mmbando BP, Drakeley CJ, Jones C, Akida J, Savaeli ZX, Kitua AY, Lemnge MM, Theander TG: Malaria morbidity and immunity among residents of villages with different Plasmodium falciparum transmission intensity in NorthEastern Tanzania. Malar J 2004, 3:26.

20. Bodker R, Akida J, Shayo D, Kisinza W, Msangeni HA, Pedersen EM, Lindsay SW: Relationship between altitude and intensity of malaria transmission in the Usambara Mountains, Tanzania. J Med Entomol 2003, 40:706-7I7.

21. Schellenberg D, Schellenberg JR, Mushi A, Savigny D, Mgalula L, Mbuya C, Victora CG: The silent burden of anaemia in Tanzanian chil- 
dren: a community-based study. Bull World Health Organ 2003, 8I:58I-590.

22. Polley SD, Mwangi T, Kocken CH, Thomas AW, Dutta S, Lanar DE, E R, Ross A, G W, Mwanakasale V, G M, B. L, J.D. C, Marsh K: Human antibodies to recombinant protein constructs of Plasmodium falciparum Apical Membrane Antigen I (AMA I) and their associations with protection from malaria. Vaccine 2004, 23:7| $8-728$

23. MacDonald: The epidemiology and control of malaria London, Oxford University Press; 1957.

24. Theisen M, Soe S, Jessing SG, Okkels LM, Danielsen S, Oeuvray C, Druilhe $P$, Jepsen $S$ : Identification of a major B-cell epitope of the Plasmodium falciparum glutamate-rich protein (GLURP), targeted by human antibodies mediating parasite killing. Vaccine 2000, 19:204-212.

Publish with Bio Med Central and every scientist can read your work free of charge

"BioMed Central will be the most significant development for disseminating the results of biomedical research in our lifetime. "

Sir Paul Nurse, Cancer Research UK

Your research papers will be:

- available free of charge to the entire biomedical community

- peer reviewed and published immediately upon acceptance

- cited in PubMed and archived on PubMed Central

- yours - you keep the copyright

Submit your manuscript here:

http://www.biomedcentral.com/info/publishing_adv.asp 